\title{
Use of a contact microphone for high-quality recording of snoring during natural or drug-induced sleep*
}

Thomas Jacques', George Korres', Nicolas Tatlas², Stylianos Potirakis², Bhik Kotecha'

'Royal National Throat, Nose and Ear Hospital, London, United Kingdom

2 Piraeus University of Applied Sciences, Greece
Rhinology Online, Vol 1: 201 - 203, 2018

http://doi.org/10.4193/RHINOL/18.029

* Received for publication:

June 27, 2018

Accepted: November 9, 2018

\begin{abstract}
Background: Selected adult patients with obstructive sleep apnoea-hypopnea syndrome (OSAHS) may benefit from surgical intervention. At present, the dominant technique for the diagnosis of the anatomical locus of upper airway obstruction during sleep is drug-induced sedation endoscopy (DISE). This technique is widespread, but has generated controversy related to its artificiality.
\end{abstract}

Methodology: We present a technique for the acquisition of high-quality and consistent recordings of snoring sounds, suitable for advanced acoustic analysis and correlation with the findings of DISE.

Results: Analysis of the generated recordings demonstrates distinct frequency-domain characteristics of snoring sounds generated by distinct anatomical subsites, for example the soft palate, or tongue base.

Conclusions: Supplemental information may potentially be obtained from the waveform of snoring sounds produced by the patient; however, no robust quantitative "assay" has been developed or been clinically validated. This may in part be related to inconsistencies or insufficient quality of the sound data acquired. This novel technique may circumvent these difficulties and permit more advanced analysis techniques with potential clinical applicability.

Key words: snoring, sleep apnoea, endoscopy, acoustic analysis, DISE

\section{Introduction}

Snoring is a widespread clinical problem, and part of the spectrum of obstructive sleep apnea hypopnea syndrome (OSAHS). Surgical and non-operative interventions for snoring and OSAHS can potentially be targeted using drug-induced sedation endoscopy (DISE), which is a dynamic investigation that can identify the site and pattern of pharyngeal collapse responsible for snoring sound production ${ }^{(1)}$. DISE remains controversial due to its limitations, particularly inconsistencies in anaesthetic protocol, classification and scoring of finding, the fact that drug-induced sedation is only an analogue of natural sleep, and inter-observer variability. Novel techniques for the investigation of snoring, particularly those that improve the level of objectivity, would therefore be valuable in both clinical and research settings.
Detailed analysis of snoring sounds may potentially allow "acoustic fingerprinting", and correlation with underlying anatomical sites of airway obstruction during sleep. Authors have explored this possibility using non-contact environmental microphones in the past ${ }^{(2-4)}$. Several basic acoustic parameters have been correlated with the presence of obstruction at either the palate or the tongue base. finding that the centre frequency of palatal snoring was lower than that of tongue base snoring (2). Possible sources of error included inconsistency in microphone and patient positioning, and the presence and variability of background noise. The use of an environmental microphone by this and other groups meant that the technique was not immediately transferable to the home environment. 


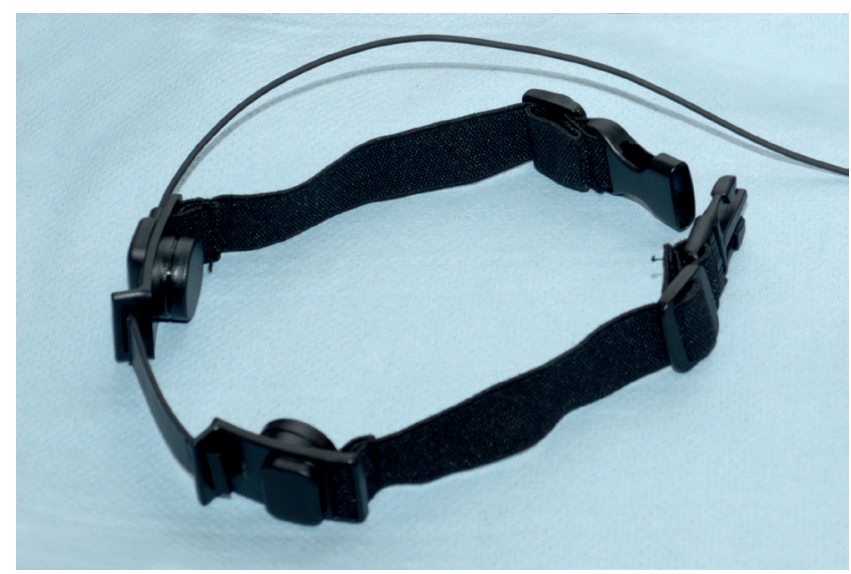

Figure 1. Contact microphone (CTH100, Clockaudio Ltd., Waterlooville, UK), utilized for high signal-to-noise ratio clinical recordings of snoring audio data.

An ideal implementation of this technique would allow its use both during sedation endoscopy (to allow the evaluation of the effect of a jaw thrust), and also during natural sleep, as part of a domiciliary polysomnogram. It therefore must be simple to apply, with standardized microphone positioning and inherent rejection of background noise, in order to produce consistent, detailed recordings that are comparable between patients.

The use of contact throat microphones (also known as laryngophones) is commonplace in environments where high-quality transmission of quiet voice is necessary, especially in environments with high-intensity background noise, in particular military environments. Laryngophones are inexpensive, robust, and can be worn around the neck on an adjustable elasticated strap. They inherently provide a very high signal-to-noise ratio, and therefore potentially represent a simple method of creating reproducible snoring sound recordings in the clinical and home settings.

\section{Materials and Methods}

We have conducted recordings of snoring sounds under a standard drug-induced sedation endoscopy protocol, in over 35 patients, as part of a pilot observational study. All patients undergoing DISE were considered eligible for inclusion. Patients were recruited in a sequential non-randomised fashion, and informed consent was obtained in all cases. Patients are anaesthetised with bolus administration of midazolam ( $2 \mathrm{mg}$ ) and propofol (1-2 $\mathrm{mg} / \mathrm{kg})$, and examined with a $4 \mathrm{~mm}$ nasendoscope by the senior author. We utilise a two-channel technique, with a contact throat microphone (Figure 1) (CTH100, Clockaudio Ltd., Waterlooville, UK), positioned adjacent to the patient's thyroid cartilage (Figure 2), and a high-quality balanced environmental microphone positioned at a standardised distance from the patient. 24-bit, $96 \mathrm{kHz}$ recordings are created digitally via a USB-
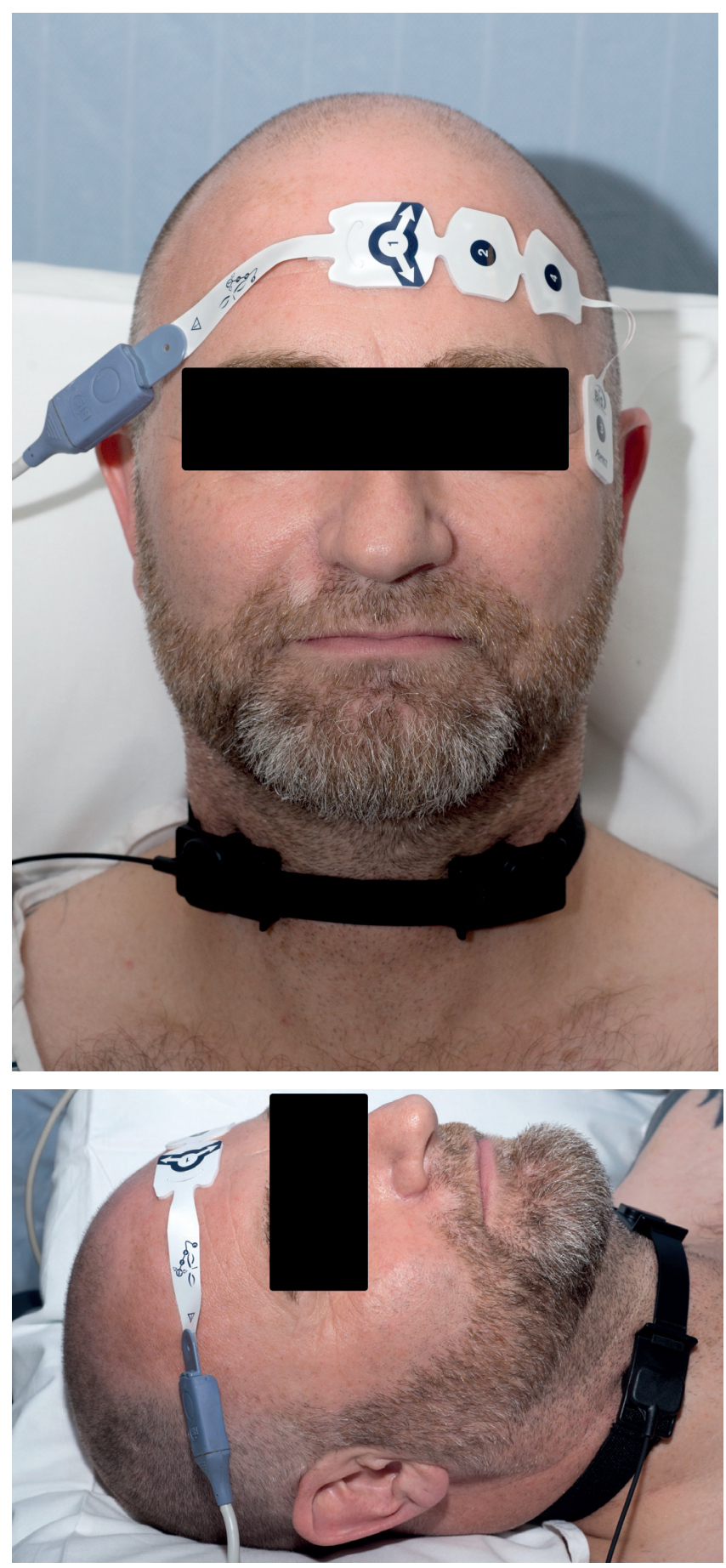

Figures 2a, 2b. Patient prepared for DISE with throat microphone in situ (also note depth-of-sedation monitoring in use).

powered analogue-digital converter, using Wavelab 6 (Steinberg Media Technologies GmbH, Germany) (Figure 3). Each patient's snoring was recorded for a minimum of five snoring cycles, in a supine position without airway manoeuvres applied.

\section{Results}

Recordings were obtained successfully from 35 patients ( 8 fe- 


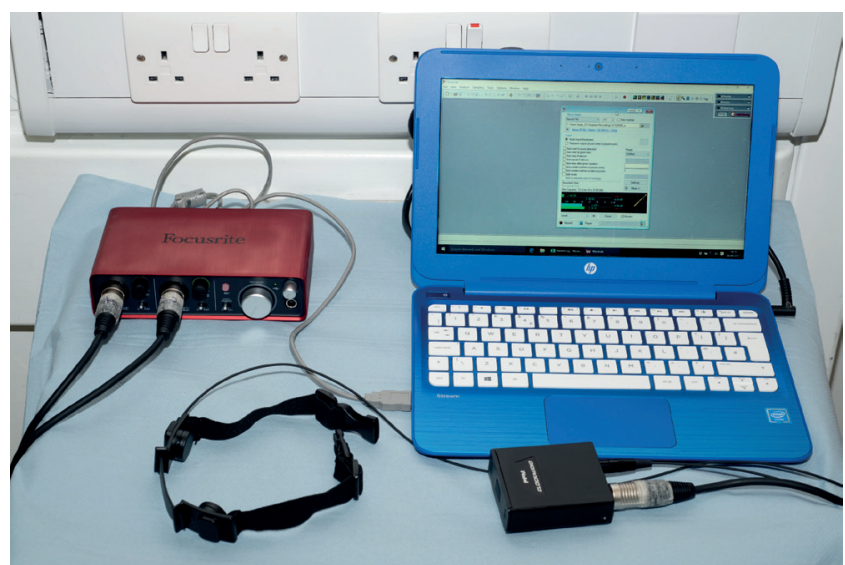

Figure 3. External USB-driven mixer, audio recording software and XLRconnected microphone and belt-pack.

male, 27 male; median age 45). Analysis of the generated recordings demonstrates distinct frequency-domain characteristics of snoring sounds generated by distinct anatomical subsites, for example the soft palate, or tongue base. In particular, the high quality of the recorded sounds allows the Tsallis entropy value of the waveforms to be calculated, along with other metrics of variability/entropy. Preliminary results suggest a relationship between Tsallis entropy and the anatomical locus of snoring as observed during the patient's DISE procedure.

No safety-related incidents or concerns were generated by the use of the contact microphone. Patient-reported tolerability of the microphone was high, with no patients reporting any discomfort related to its placement. Tolerability of the microphone during natural sleep has not yet been investigated but will be examined in future research.

\section{Discussion}

There are several potential benefits of this novel technique. The simplicity of using the throat microphone makes it possible to integrate the recording method, if validated, into the existing protocol for limited polysomnography. This would add more useful data to the output of a home sleep study, potentially even obviating the need to perform more invasive testing in straightforward cases. The higher quality of recordings will allow waveform analysis to be carried out, drawing more detailed parallels between the acoustic signal from snoring and the anatomical site of airway obstruction in sleep. Further work is recommended to expand the library of snoring sounds from a larger number of patients, to allow statistical correlation between the spectral analysis of the obtained snoring audio and the results of DISE.

\section{Acknowledgement}

The study received no external funding.

\section{Authorship contribution}

TJ: Study formulation, data collection, manuscript preparation; GK: Study formulation, technical preparation; NT: Technical preparation, data analysis; SP: Technical preparation, data analysis; BK: Study formulation, data collection, manuscript preparation.

\section{Conflict of interest}

The authors declare no conflict of interest.
1. De Vito A, Carrasco Llatas M, Vanni A, Bosi M, Braghiroli A, Campanini A, de Vries N Hamans E, Hohenhorst W, Kotecha BT, Maurer J, Montevecchi F, Piccin O, Sorrenti G, Vanderveken OM, Vicini C. European position paper on drug-induced sedation endoscopy (DISE). Sleep Breath 2014; 18 453-65.

2. Quinn SJ, Huang L, Ellis PD, Williams JE. The differentiation of snoring mechanisms using sound analysis. Clin Otolaryngol Allied Sci 1996; 21: 119-123.

3. Osborne JE, Osman EZ, Hill PD, Lee BV Sparkes C. A new acoustic method of differentiating palatal from non-palatal snoring. Clin Otolaryngol Allied Sci 1999; 24
130-133.

4. Saunders NC, Tassone P, Wood G, Norris A, Harries M, Kotecha B. Is acoustic analysis of snoring an alternative to sleep nasendoscopy? Clin Otolaryngol Allied Sci 2004; 29: 242-246.
Professor Bhik Kotecha FRCS MPhil Consultant Otolaryngologist Head and Neck Surgeon Royal National Throat, Nose and Ear Hospital

London

United Kingdom

Tel: +44-20-7915 1313

E-mail: bhik.kotecha@nhs.net 